\title{
Fault Detection for Quantized Networked Control Systems
}

\author{
Wei-Wei Che, ${ }^{1}$ Zhi-Yong Mei, $^{2}$ and Yu-Long Wang ${ }^{3}$ \\ ${ }^{1}$ Key Laboratory of Manufacturing Industrial Integrated Automation, Shenyang University, Shenyang 110044, China \\ ${ }^{2}$ Institute of Information, Shenyang University, Shenyang 110044, China \\ ${ }^{3}$ School of Electronics and Information, University of Science and Technology, Zhenjiang 212003, China
}

Correspondence should be addressed to Wei-Wei Che; cwwemail1980@126.com

Received 21 August 2013; Accepted 5 October 2013

Academic Editor: Tao Li

Copyright (C) 2013 Wei-Wei Che et al. This is an open access article distributed under the Creative Commons Attribution License, which permits unrestricted use, distribution, and reproduction in any medium, provided the original work is properly cited.

\begin{abstract}
The fault detection problem in the finite frequency domain for networked control systems with signal quantization is considered. With the logarithmic quantizer consideration, a quantized fault detection observer is designed by employing a performance index which is used to increase the fault sensitivity in finite frequency domain. The quantized measurement signals are dealt with by utilizing the sector bound method, in which the quantization error is treated as sector-bounded uncertainty. By using the KalmanYakubovich-Popov (GKYP) Lemma, an iterative LMI-based optimization algorithm is developed for designing the quantized fault detection observer. And a numerical example is given to illustrate the effectiveness of the proposed method.
\end{abstract}

\section{Introduction}

Recently, due to many advantages of networked control systems, such as lower cost, easier installation and maintenance, and higher reliability, NCSs have been found successfully industrial applications in automobiles, manufacturing plants, aircraft, HVAC, systems and unmanned vehicles. However, the insertion of the communication channels results in discrepancies between the data information to be transmitted and their associated remotely transmitted images, hence raising new interesting and challenging problems such as quantization, packet losses, and time delays. As is well known to all, quantization always exists in bandwidth limited networked systems and the performance of NCSs will be inevitably subject to the effect of quantization error. Hence, the quantization problem of NCSs has long been studied and many important results have been reported in [1-10] and the references therein. Two most pertinent references to this paper are the work [2] and the following work [3]. In [2], the problem of quadratic stabilization of discretetime single-input single-output (SISO) linear time-invariant systems using quantized feedback is studied. In [3], the work of [2] is generalized to general multi-input multioutput (MIMO) systems and to control problems requiring performances. This is done using the so-called sector bound method, which is based on using a simple sector bound to model the quantization error. This method has been employed by quite a few researchers and many results have been given correspondingly [4-7] and so on.

On the other hand, fault detection (FD) is a very significant problem and has attracted a lot of attention in the past two decades. A fault is defined as an unpermitted deviation of at least one characteristic property of a variable from an acceptable behaviour. Such a fault disturbs the normal operation of an automatic system, thus causing an unacceptable deterioration of the performance of the system $[11,12]$. To detect the fault, an observer is usually designed which generates the residual signal, and, by satisfying certain performances, the observer parameters are then determined. Up to now, the studies on the FD are mainly categorized into two classes depending on the fault frequency domain. There are many results that study the FD problem in the full frequency domain, such as $[13,14]$. Recently, there are many studies considering the fault in finite frequency domain which much more accords with practice. Because in practice, faults are usually in the finite frequency domain; for example, for an incipient fault signal, the fault information is contained within a low frequency band as the fault development is slow as stated in [15]. Another important finite frequency fault is the actuator stuck fault whose frequency is zero. The stuck 
fault occurs when an aircraft control surface (such as the rudder or an aileron) is stuck at some fixed value as stated in [16]. And the stuck fault is also considered for the F16 aircraft in [17]. So, the finite frequency domain method to FD has been paid more attention to many new results occur successively [18-20]. In networked control systems, FD problem also exists and is unavoidably. So far, there have been some studies on the FD problem of the networked control systems $[21,22]$. But to the best of the authors' knowledge, there has been no work considering the quantized FD problem in finite frequency domain for networked control systems.

Motivated by the above-mentioned reason, in this paper, the quantized FD observer design problem for networked control systems with logarithmic quantizers is studied. The quantization errors are modeled as sector-bounded uncertainties. By employing the GKYP method, a quantized FD observer design method is proposed with an iterative LMIbased optimization algorithm. Finally, a numerical example is given to show the effectiveness of the proposed method.

The organization of this paper is as follows. Section 2 presents the problem under consideration and some preliminaries. Section 3 gives design methods of quantized FD observer design strategies. In Section 4, an example is presented to illustrate the effectiveness of the proposed methods. Finally, Section 5 gives some concluding remarks.

Notation. For a matrix $E, E^{T}, E^{\perp}$, and $E^{*}$ denote its transpose, orthogonal complement, and complex conjugate transpose, respectively. And $A^{\dagger}=A^{T}\left(A A^{T}\right)^{-1}$ denotes its MoorePenrose inverse. I denotes the identity matrix with an appropriate dimension. For a symmetric matrix, $A>(\geq) 0$ and $A<(\leq) 0$ denote positive (semi-) definiteness and negative (semi-) definiteness. The Hermitian part of a square matrix $M$ is denoted by $\mathrm{He}(M):=M+M^{*}$. The symbol $H_{n}$ stands for the set of $n \times n$ Hermitian matrices. The symbol “*” within a matrix represents the symmetric entries. $\sigma_{\max }(G)$ and $\sigma_{\min }(G)$ denote maximum and minimum singular value of the transfer matrix $G$, respectively.

\section{Problem Statement and Preliminaries}

2.1. Problem Statement. Consider an LTI discrete-time system as

$$
\begin{gathered}
x(k+1)=A x(k)+B_{1} f(k)+B_{2} u_{c}(k), \\
y(k)=C x(k)+D_{1} f(k),
\end{gathered}
$$

where $x(k) \in R^{n}$ is the state, $u_{c}(k) \in R^{u}$ is the control input, and $f(k) \in R^{f}$ is the fault input vector, respectively. $A, B_{1}$, $B_{2}, C$, and $D_{1}$ are known constant matrices of appropriate dimensions. Without loss of generality, assume that $(A, C)$ is observable and $B_{1}$ is of full column rank.

To formulate the quantized FD problem, consider the quantized FD observer with the following form.
Consider a dynamic observer-based control strategy for (1) with observer given by

$$
\begin{gathered}
\widehat{x}(k+1)=A \hat{x}(k)+B_{2} u_{c}(k)+L\left(y_{c}(k)-\bar{y}_{c}(k)\right) \\
\bar{y}_{c}(k)=C \hat{x}(k), \\
u_{c}(k)=K \hat{x}(k), \\
r(k)=y_{c}(k)-\bar{y}_{c}(k),
\end{gathered}
$$

where $\bar{y}_{c}(k) \in R^{y}$ is the observer output, $\widehat{x}(k) \in R^{n}$ is the state estimation of system (1), and $r(k) \in R^{r}$ is the residual signal. $L$ is the observer gain to be designed. Due to the insertion of the communication channel, the measurement signals will be quantized before they are transmitted to the filter through the network. The quantizer is denoted as $q[\cdot]=$ $\left[q_{1}[\cdot], q_{2}[\cdot], \ldots, q_{y}[\cdot]\right]^{T}$, which is assumed to be symmetric; that is, $q_{j}[-\varepsilon]=-q_{j}[\varepsilon], j=1,2, \ldots, y$. In this paper, the quantizer is selected as a logarithmic one, and, for each $q_{j}[\cdot]$, the quantization levels are given by

$$
\begin{array}{r}
V_{j}=\left\{ \pm v_{i}^{(j)}: v_{i}^{(j)}=\rho_{j}^{i} v_{0}^{(j)}, i=\right. \\
\quad, \pm 1, \pm 2, \ldots\} \cup\{0\} \\
v_{0}^{(j)}>0,0<\rho_{j}<1 .
\end{array}
$$

As in $[3,4]$, the associated quantizer is defined as follows:

$$
\begin{aligned}
q_{j}[\varepsilon]= \begin{cases}\rho_{j}^{i} v_{0}^{j}, & \text { if } \frac{1}{1+\delta_{j}} \rho_{j}^{i} v_{0}^{j}<\varepsilon<\frac{1}{1-\delta_{j}} \rho_{j}^{i} v_{0}^{j}, \varepsilon>0 \\
0, & \text { if } \varepsilon=0 \\
-q_{j}[-\varepsilon], & \text { if } \varepsilon<0,\end{cases} \\
\quad \delta_{j}=\frac{1-\rho_{j}}{1+\rho_{j}} .
\end{aligned}
$$

Then, based on the quantizer (4), the measurement signal at the filter end is with the form as

$$
y_{c}(k)=q(y(k))=\left[\begin{array}{c}
q_{1}\left(y_{1}(k)\right) \\
\vdots \\
q_{y}\left(y_{y}(k)\right)
\end{array}\right]=(I+\Delta(k)) y(k),
$$

where

$$
\begin{array}{r}
\Delta(k)=\operatorname{diag}\left\{\Delta_{1}(k), \Delta_{2}(k), \ldots, \Delta_{y}(k)\right\}, \\
\Delta_{i}(k) \in\left[-\delta_{i}, \delta_{i}\right] .
\end{array}
$$

Combining FD observer (2) with system (1) and the quantized measurement (6), the following quantized error dynamic system is obtained:

$$
\begin{aligned}
& \begin{array}{l}
x(k+1)=\left(A+B_{2} K\right) x(k)+B_{1} f(k)-B_{2} K e(k), \\
e(k+1)=(A-L C) e(k)-L \Delta(k) C x(k)
\end{array} \\
& \quad+\left(B_{1}-L \Delta(k) D_{1}\right) f(k), \\
& r(k)=C e(k)+\Delta(k) C x(k)+(I+\Delta(k)) D_{1} f(k),
\end{aligned}
$$

where $e(k)=x(k)-\widehat{x}(k)$. 
Facilitating the presentation, (8) can be rewritten as

$$
\begin{gathered}
x_{e}(k+1)=\bar{A} x_{e}(k)+\bar{B}_{1} f(k) \\
r(k)=\bar{C} x_{e}(k)+\bar{D}_{1} f(k),
\end{gathered}
$$

where $x_{e}(k)=\left[\begin{array}{l}e(k) \\ x(k)\end{array}\right]$ and

$$
\begin{aligned}
& \bar{A}=\left[\begin{array}{cc}
A-L C & -L \Delta(k) C \\
-B_{2} K & A+B_{2} K
\end{array}\right], \\
& \bar{B}_{1}=\left[\begin{array}{c}
B_{1}-L(I+\Delta(k)) D_{1} \\
B_{1}
\end{array}\right] \text {, }
\end{aligned}
$$

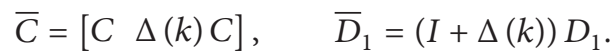

Note that the dynamics of the residual signal depends on the fault $f(k)$, to detect the fault effects; quantized observer (2) is designed in this work such that the following conditions are satisfied:

$$
\begin{aligned}
& \text { (i) }: \bar{A}=\left[\begin{array}{cc}
A-L C & -L \Delta(k) C \\
-B_{2} K & A+B_{2} K
\end{array}\right] \text { is stable, } \\
& \text { (ii) }: \inf \sigma_{\min }\left(G_{r f}\left(e^{j \theta}\right)\right)>\gamma, \quad \forall \theta \in\left[v_{1}, v_{2}\right],
\end{aligned}
$$

where

$$
G_{r f}\left(e^{j \theta}\right):=\bar{C}\left(e^{j \theta} I-\bar{A}\right)^{-1}\left(\bar{B}_{1}-L \bar{D}_{1}\right)+\bar{D}_{1} .
$$

Remark 1. Condition (ii) is a finite frequency performance index used to increase the fault sensitivity. Note that $v_{1}, v_{2}$ are given scalars which reflects the frequency range of faults.

The problem addressed in this paper is as follows.

Quantized FD Control Problem. Considering the effects of the quantization, design a quantized FD observer such that the error system (8) is with high fault sensitivity in finite frequency domain.

2.2. Preliminaries. The following lemma presented will be used in this paper.

Lemma 2 (see [23]). Consider a transfer function matrix $G\left(e^{j \theta}\right):=C\left(e^{j \theta} I-A\right)^{-1} B+D$; let a symmetric matrix $\Pi$ and scalars $\nu_{1}, \nu_{2}$ be given; the following statements are equivalent.

(i) The finite frequency inequality

$$
\left[\begin{array}{c}
G\left(e^{j \theta}\right) \\
I
\end{array}\right]^{*} \Pi\left[\begin{array}{c}
G\left(e^{j \theta}\right) \\
I
\end{array}\right]<0, \quad \forall \theta \in\left[\nu_{1}, \nu_{2}\right] .
$$

(ii) There exist matrices $P, Q \in \mathbf{H}_{n}$ of appropriate dimensions, satisfying $Q>0$, and

$$
\left[\begin{array}{cc}
A & B \\
I & 0
\end{array}\right]^{*} \Xi\left[\begin{array}{cc}
A & B \\
I & 0
\end{array}\right]+\left[\begin{array}{cc}
C & D \\
0 & I
\end{array}\right]^{*} \Pi\left[\begin{array}{cc}
C & D \\
0 & I
\end{array}\right]<0
$$

where

$$
\Xi=\left[\begin{array}{cc}
-P & e^{j\left(v_{1}+v_{2}\right) / 2} Q \\
e^{-j\left(\nu_{1}+v_{2}\right) / 2} Q & P-\left(2 \cos \left(\left(\nu_{2}-v_{1}\right) / 2\right)\right) Q
\end{array}\right] .
$$

Lemma 3 (Finsler's Lemma). Let $x \in R^{n}, Q \in R^{n \times n}$, and $U \in$ $R^{n \times m}$. Let $U^{\perp}$ be any matrix such that $U^{\perp} U=0$. The following statements are equivalent:

(i) $x^{*} Q x<0$, for all $U^{*} x=0, x \neq 0$,

(ii) $U^{\perp} Q U^{\perp^{*}}=0$,

(iii) $\exists \mu \in R: Q-\mu U U^{*}<0$,

(iv) $\exists y \in R^{m \times n}: Q+U Y+Y^{*} U^{*}<0$.

Lemma 4 (Projection Lemma). Let $\Gamma, \Lambda, \Theta$ and be given. There exists a matrix $F$ satisfying $\Gamma F \Lambda+(\Gamma F \Lambda)^{T}+\Theta<0$ if and only if the following two conditions hold:

$$
\Gamma^{\perp} \Theta<0, \quad \Lambda^{T^{\perp}} \Theta \Lambda^{T^{\perp^{T}}}<0 .
$$

Lemma 5 (see [24]). For any real matrices $Y, M, F$, and $E$ with compatible dimensions and $F^{T} F \leq \delta^{2} I$, where $\delta>0$ is a scalar, then

$$
Y+M F E+(M F E)^{T}<0
$$

holds if and only if there exists a scalar $\varepsilon>0$, such that

$$
Y+\frac{1}{\varepsilon} M M^{T}+\varepsilon \delta^{2} E^{T} E<0 .
$$

\section{Quantized FD Observer Design}

In this section, an inequality for the stability condition (i) is given first. Then, considering the fault sensitivity problem, an inequality is given for the fault sensitivity condition (ii).

Firstly, considering the stability condition (i), we have the following lemma.

Lemma 6. Consider system (9) if there exists a matrix $\bar{X}=$ $\left[\begin{array}{ll}X & 0 \\ 0 & X\end{array}\right]>0$ such that the following inequality holds:

$$
\left[\begin{array}{cccccc}
-X & * & * & * & * & * \\
0 & -X & * & * & * & * \\
X A-\Gamma^{T} C & 0 & -X & * & * & * \\
-X B_{2} K & X A+X B_{2} K & 0 & -X & * & * \\
0 & 0 & -\Gamma & 0 & -\varepsilon I & * \\
0 & \delta_{a} \varepsilon C & 0 & 0 & 0 & -\varepsilon I
\end{array}\right]<0
$$

where $\Gamma=L^{T} X$

Proof. It is easy to know that (i) holds if there exists $\bar{X}>0$, such that

$$
\bar{A}^{T} \bar{X} \bar{A}-\bar{X}<0 \text {. }
$$

By using the Schur complement lemma, we have that

$$
\left[\begin{array}{cc}
-\bar{X} & \bar{A}^{T} \bar{X} \\
\bar{X} \bar{A} & -\bar{X}
\end{array}\right]<0 .
$$


Equation (21) can be converted into

$$
\left[\begin{array}{cccccc}
-X & * & * & * & * & * \\
0 & -X & * & * & * & * \\
X A-\Gamma^{T} C & -X L \Delta(k) C & -X & * & * & * \\
-X B_{2} K & X A+X B_{2} K & 0 & -X & * & * \\
0 & 0 & -\Gamma & 0 & -\varepsilon I & * \\
0 & \delta_{a} \varepsilon C & 0 & 0 & 0 & -\varepsilon I
\end{array}\right]<0 .
$$

Obviously, (22) can be rewritten as

$$
\begin{aligned}
& {\left[\begin{array}{cccccc}
-X & * & * & * & * & * \\
0 & -X & * & * & * & * \\
X A-\Gamma^{T} C & 0 & -X & * & * & * \\
-X B_{2} K & X A+X B_{2} K & 0 & -X & * & * \\
0 & 0 & -\Gamma & 0 & -\varepsilon I & * \\
0 & \delta_{a} \varepsilon C & 0 & 0 & 0 & -\varepsilon I
\end{array}\right]} \\
& +\left[\begin{array}{c}
0 \\
0 \\
-\Gamma \\
0
\end{array}\right] \Delta(k)\left[\begin{array}{llll}
0 & C & 0 & 0
\end{array}\right] \\
& \left.+\left[\begin{array}{c}
0 \\
0 \\
-\Gamma \\
0
\end{array}\right] \Delta(k)\left[\begin{array}{llll}
0 & C & 0 & 0
\end{array}\right]\right]^{T}<0 .
\end{aligned}
$$

By using Lemma 5, (19) holds, which shows that condition (i) holds if (19) holds. This completes the proof

In the following, the fault sensitivity problem is studied. Considering system (9), the following lemma is presented to give the fault sensitivity condition.

Lemma 7. Let real matrix $\bar{A}, \bar{B}_{1}, \bar{C}, \bar{D}_{1}$, a symmetric matrix $\Pi=\left[\begin{array}{cc}-I & 0 \\ 0 & \gamma^{2} I\end{array}\right]$, and scalars $\vartheta_{1}, \vartheta_{2}$ be given; consider system (9), then the following conditions are equivalent.

(i) The following finite frequency inequality

$$
\sigma_{\min }\left(G_{r f}\left(e^{j \theta}\right)\right)>\gamma, \quad \forall \theta \in\left[\vartheta_{1}, \vartheta_{2}\right]
$$

holds, where $G_{r f}\left(e^{j \theta}\right)=\bar{C}\left(e^{j \theta} I-\bar{A}\right)^{-1} \bar{B}_{1}+\bar{D}_{1}$ is the transfer function matrix from $f(k)$ to $r(k)$.

(ii) There exist $2 n \times 2 n$ Hermitian matrices $P$ and $Q$ satisfying $Q>0$, and

$$
\left[\begin{array}{cc}
\bar{A} & \bar{B} \\
I & 0
\end{array}\right]^{*} \Xi\left[\begin{array}{cc}
\bar{A} & \bar{B}_{1} \\
I & 0
\end{array}\right]+\left[\begin{array}{cc}
\bar{C} & \bar{D}_{1} \\
0 & I
\end{array}\right]^{*} \Pi\left[\begin{array}{cc}
\bar{C} & \bar{D}_{1} \\
0 & I
\end{array}\right]<0,
$$

where

$$
\Xi=\left[\begin{array}{cc}
-P & e^{j\left(v_{1}+v_{1}\right) / 2} Q \\
e^{-j\left(v_{1}+v_{1}\right) / 2} Q & P-\left(2 \cos \frac{\nu_{1}-v_{1}}{2}\right) Q
\end{array}\right] .
$$

Note that condition (25) in Lemma 7 can be rewritten as

$$
\left[\Upsilon \begin{array}{ll}
\Upsilon & I
\end{array}\right] T\left[\begin{array}{cc}
\Xi & 0 \\
0 & \Pi
\end{array}\right] T^{*}[\Upsilon \quad I]^{*}<0
$$

where

$$
\Upsilon=\left[\begin{array}{ccc}
A^{T}-C^{T} L^{T} & -K^{T} B_{2}^{T} & C^{T} \\
-C^{T} \Delta(k) L^{T} & A^{T}+K^{T} B_{2}^{T} & \Delta(k) C^{T} \\
B_{1}^{T}-D_{1}^{T}(I+\Delta(k))^{T} L^{T} & B_{1}^{T} & D_{1}^{T}(I+\Delta(k))^{T}
\end{array}\right],
$$

and $T$ is the permutation matrix defined as

$$
\left[\begin{array}{llllll}
N_{1} & N_{2} & N_{3} & N_{4} & N_{5} & N_{6}
\end{array}\right] T=\left[\begin{array}{llllll}
N_{1} & N_{2} & N_{5} & N_{3} & N_{4} & N_{6}
\end{array}\right] \text {. }
$$

By using Lemma 3, we have that (27) is equivalent to the existence of a multiplier $\mathscr{X}$ such that

$$
T\left[\begin{array}{cc}
\Xi & 0 \\
0 & \Pi
\end{array}\right] T^{*}<H e\left[\begin{array}{c}
I \\
\Upsilon
\end{array}\right] \mathscr{X} .
$$

Obviously, $\Upsilon$ can be rewritten as

$$
\begin{aligned}
\Upsilon= & {\left[\begin{array}{ccc}
A^{T} & -K^{T} B_{2}^{T} & C^{T} \\
0 & A^{T}+K^{T} B_{2}^{T} & \Delta(k) C^{T} \\
B_{1}^{T} & B_{1}^{T} & D_{1}^{T}(I+\Delta(k))^{T}
\end{array}\right] } \\
& +\left[\begin{array}{c}
-C^{T} \\
-C^{T} \Delta(k) \\
-D_{1}^{T}(I+\Delta(k))^{T}
\end{array}\right] L^{T}\left[\begin{array}{lll}
I & 0 & 0
\end{array}\right]=\mathscr{A}+\mathscr{B} L^{T} \mathscr{C} .
\end{aligned}
$$

To facilitate dealing with the problem, restrict $\mathscr{X}$ with the following structure:

$$
\mathscr{X}=\left\{\mathscr{C}^{\dagger} X R+\left(I-\mathscr{C}^{\dagger} \mathscr{C}\right) V \mid X \in \mathbb{R}^{n \times n}, \operatorname{det} X \neq 0\right\},
$$

where $R$ is a matrix to be specified later, $X, V$ are matrix variables.

Then, we have

$$
\begin{aligned}
\Upsilon \mathscr{X} & =\left(\mathscr{A}+\mathscr{B} L^{T} \mathscr{C}\right)\left(\mathscr{C}^{\dagger} X R+\left(I-\mathscr{C}^{\dagger} \mathscr{C}\right) V\right) \\
& =\mathscr{A} \mathscr{X}+\mathscr{B} \Gamma R .
\end{aligned}
$$

Then the following lemma is given to show that the restriction of $\Upsilon \mathscr{X}$ does not introduce conservatism if the matrix $R$ is specified appropriately.

Lemma 8. Consider system (9), let $R$ and $\Pi$ be with appropriate dimensions, and let $Q>0$; then the following statements are equivalent.

(i) There exists a gain matrix L such that condition (25) and

$$
\left(S R^{*}\right)^{\perp} S T\left[\begin{array}{cc}
\Xi & 0 \\
0 & \Pi
\end{array}\right] T^{*} S^{*}\left(S R^{*}\right)^{\perp *}<0
$$

hold, where

$$
S=\left[\begin{array}{cccccc}
A^{T}-C^{T} L^{T} & -K^{T} B_{2}^{T} & C^{T} & I & 0 & 0 \\
-C^{T} \Delta(k) L^{T} & A^{T}+K^{T} B_{2}^{T} & \Delta(k) C^{T} & 0 & I & 0 \\
B_{1}^{T}-D_{1}^{T}(I+\Delta(k))^{T} L^{T} & B_{1}^{T} & D_{1}^{T}(I+\Delta(k))^{T} & 0 & 0 & I \\
I & 0 & 0 & 0 & 0 & 0 \\
0 & I & 0 & 0 & 0 & 0
\end{array}\right] .
$$

(ii) There exists matrix variable $\Gamma=L^{T} X$ such that

$$
T\left[\begin{array}{cc}
\Xi & 0 \\
0 & \Pi
\end{array}\right] T^{*}<H e\left[\begin{array}{c}
\mathscr{X} \\
-\mathscr{A} \mathscr{X}-\mathscr{B} \Gamma R
\end{array}\right] .
$$


Proof. The proof is similar to the proof of Lemma 5 in [20], it is omitted here.

Then, combining Lemmas 7 and 8, the following theorem is presented.

Denote

$$
\begin{aligned}
& F_{a 1}=\left[\begin{array}{cccc}
0 & 0 & 0 & 0 \\
0 & 0 & 0 & 0 \\
0 & 0 & 0 & 0 \\
0 & 0 & 0 & 0 \\
I & -C^{T} & 0 & 0 \\
0 & 0 & -D_{1}^{T} & -D_{1}^{T}
\end{array}\right] \\
& F_{a 2}=\left[\begin{array}{cccccc}
C^{T} V_{c 1} & C^{T} V_{c 2} & C^{T} V_{c 3} & C^{T} V_{c 4} & C^{T} V_{c 5} & C^{T} V_{c 6} \\
\Gamma & \Gamma & 0 & 0 & \Gamma & -\Gamma B_{1} \\
V_{c 1} & V_{c 2} & V_{c 3} & V_{c 4} & V_{c 5} & V_{c 6} \\
\Gamma & \Gamma & 0 & 0 & \Gamma & -\Gamma B_{1}
\end{array}\right], \\
& \Lambda=\left[\begin{array}{cccccc}
-P_{11}-X-X^{T} & * & * & * & * & * \\
-P_{21}-V_{b 1}-X^{T} & -P_{22}-V_{b 2}-V_{b 2}^{T} & * & * & * & * \\
e^{-j 9_{c}} Q_{11}-V_{c 1} & e^{-j \vartheta_{c}} Q_{12}-V_{c 2} V_{b 3}^{T} & \Phi & * & * & * \\
\Phi_{1} & \Phi_{2} & \Phi_{3} & \Phi_{4} & * & * \\
\Phi_{5} & \Phi_{6} & \Phi_{7} & \Phi_{8} & \Phi_{9} & * \\
\Phi_{10} & \Phi_{11} & \Phi_{12} & \Phi_{13} & \Phi_{14} & \Phi_{15}
\end{array}\right]
\end{aligned}
$$

where

$$
\begin{aligned}
& \Phi=P_{12}-2 \cos \vartheta_{w} Q_{12}-V_{c 3}-V_{c 3}^{T}, \\
& \Phi_{1}=e^{-j \vartheta_{c}} Q_{12}^{T}+A^{T} X-K^{T} B_{2}^{T} V_{b 1}+C^{T} V_{c 1}-C^{T} \Gamma-X^{T}, \\
& \Phi_{2}=e^{-j \vartheta_{c}} Q_{22}^{T}+A^{T} X-K^{T} B_{2}^{T} V_{b 2}-C^{T} V_{c 3}+C^{T} \Gamma-V_{b 4}^{T}, \\
& \Phi_{3}=P_{12} 2 \cos \vartheta_{w} Q_{22}-K^{T} B_{2}^{T} V_{b 3}+C^{T} V_{3}+V_{c 4}^{T}, \\
& \Phi_{4}=-K^{T} B_{2}^{T} V_{b 4}+C^{T} V_{c 4}-\left(K^{T} B_{2}^{T} V_{b 4}-C^{T} V_{c 4}\right)^{T}, \\
& \Phi_{5}=\left(A^{T}+K^{T} B_{2}^{T}\right) V_{b 1}-X^{T}, \\
& \Phi_{6}=\left(A^{T}+K^{T} B_{2}^{T}\right) V_{b 2}-V_{b 5} \text {, } \\
& \Phi_{7}=\left(A^{T}+K^{T} B_{2}^{T}\right) V_{b 3}-V_{c 5} \text {, } \\
& \Phi_{8}=-I+\left(A^{T}+K^{T} B_{2}^{T}\right) V_{b 4}+V_{c 5}^{T}+X^{T} A-V_{b 5}^{T} B_{2} K-\Gamma^{T} C, \\
& \Phi_{9}=\left(A^{T}+K^{T} B_{2}^{T}\right) V_{b 5}-V_{b 5}^{T}\left(A+B_{2} K\right), \\
& \Phi_{10}=B_{1}^{T} X+B_{1}^{T} V_{b 1}+B_{1}^{T} X^{T}-D_{1}^{T} V_{c 1}-D_{1}^{T} \Gamma, \\
& \Phi_{11}=B_{1}^{T} X+B_{1}^{T} V_{b 2}-V_{b 6}^{T}-D_{1}^{T} V_{c 2}-D_{1}^{T} \Gamma \text {, } \\
& \Phi_{12}=B_{1}^{T} V_{b 3}-V_{c 6}-D_{1}^{T} V_{c 3} \text {, } \\
& \Phi_{13}=B_{1}^{T} V_{b 4}+\left[A^{T} X B_{1}-K^{T} B_{2}^{T} V_{b 6}\right. \\
& \left.+C^{T} V_{c 6}+C^{T} \Gamma B_{1}\right]^{T}-D_{1}^{T} V_{c 4}, \\
& \Phi_{14}=B_{1}^{T} X+B_{1}^{T} V_{b 5}+\left[A^{T}+K^{T} B_{2}^{T} V_{b 6}\right]^{T}-D_{1}^{T} V_{c 5}-D_{1}^{T} \Gamma,
\end{aligned}
$$

$$
\begin{aligned}
\Phi_{15}= & B_{1}^{T} X B_{1}-B_{1}^{T} V_{b 6}+\left[B_{1}^{T} X B_{1}-B_{1}^{T} V_{b 6}\right]^{T} \\
& -D_{1}^{T} V_{c 6}+D_{1}^{T} \Gamma B_{1},
\end{aligned}
$$

with

$$
\vartheta_{c}=\frac{\left(\vartheta_{1}+\vartheta_{2}\right)}{2}, \quad \vartheta_{\omega}=\frac{\left(\vartheta_{2}-\vartheta_{1}\right)}{2} .
$$

Theorem 9. Consider system (9); let $\gamma>0$ and $\delta_{a}>0$ be given constants and

$$
\Pi:=\left[\begin{array}{cc}
-I & 0 \\
0 & \gamma^{2} I
\end{array}\right], \quad P, Q \in H_{n}
$$

and $Q>0$. Provided that $R=\left[\begin{array}{lllll}I & I & 0 & 0 & -B_{1}\end{array}\right]$, then

$$
\sigma_{\min } G_{r f}\left(e^{j \theta}\right)>\gamma, \quad \forall \theta \in\left[\vartheta_{2}, \vartheta_{2}\right],
$$

holds if there exist matrix variables $V_{b i}, V_{c i}, i=1, \ldots, 6, X$, and scalars $\vartheta_{1}, \vartheta_{2}$, and $\varepsilon$ such that

$$
\left[\begin{array}{ccc}
\Lambda & F_{a 1} & \delta_{a} \varepsilon F_{a 2}^{T} \\
* & -\varepsilon I & 0 \\
* & * & -\varepsilon I
\end{array}\right]<0 .
$$

Proof. By Lemmas 7 and 8, we have that (42) holds if inequality (36) holds. Similar to the proof of Theorem 1 in [20], since $\mathscr{C}=\left[\begin{array}{lll}I & 0 & 0\end{array}\right]$, we know that

$$
\mathscr{C}^{\dagger}=\left[\begin{array}{l}
I \\
0 \\
0
\end{array}\right] \text {. }
$$

Then from (32) we have

$$
\mathscr{X}=\left[\begin{array}{l}
I \\
0 \\
0
\end{array}\right] X R+\left[\begin{array}{lll}
0 & 0 & 0 \\
0 & I & 0 \\
0 & 0 & I
\end{array}\right] V .
$$

Partition $V$ as $V=\left[\begin{array}{c}V_{a} \\ V_{b} \\ V_{c}\end{array}\right]$; then we get

$$
\mathscr{X}=\left[\begin{array}{c}
X R \\
V_{b} \\
V_{c}
\end{array}\right] .
$$

So, (36) can be written as

$$
\begin{aligned}
T\left[\begin{array}{cc}
\Xi & 0 \\
0 & \Pi
\end{array}\right] T^{*} & \\
<H e & {\left[\begin{array}{cccc}
I & 0 & 0 & 0 \\
0 & I & 0 & 0 \\
0 & 0 & I & 0 \\
-A^{T} & K^{T} B_{2}^{T} & -C^{T} & C^{T} \\
0 & -A^{T}-K^{T} B_{2}^{T} & -\Delta(k) C^{T} & C^{T} \Delta(k) \\
-B_{1}^{T} & -B_{1}^{T} & D_{1}^{T}(I+\Delta(k)) & D_{1}^{T}(I+\Delta(k))
\end{array}\right] } \\
& \times\left[\begin{array}{c}
X R \\
V_{b} \\
V_{c} \\
\Gamma R
\end{array}\right] .
\end{aligned}
$$


Let $V_{b}=\left[V_{b 1}-V_{b 6}\right], V_{c}=\left[V_{c 1}-V_{c 6}\right]$, and partition $P$ and $Q$ as $P=\left[\begin{array}{ll}P_{11} & P_{12} \\ P_{21} & P_{22}\end{array}\right], Q=\left[\begin{array}{ll}\mathrm{Q}_{11} & \mathrm{Q}_{12} \\ \mathrm{Q}_{12}^{T} & \mathrm{Q}_{22}\end{array}\right]>0$; then we have

$$
\begin{aligned}
\Lambda & +F_{a 1} \operatorname{diag}\{\Delta(k), \Delta(k), \Delta(k), \Delta(k)\} F_{a 2} \\
& +\left(F_{a 1} \operatorname{diag}\{\Delta(k), \Delta(k), \Delta(k), \Delta(k)\} F_{a 2}\right)^{T}<0,
\end{aligned}
$$

where $\Delta(k)$ satisfyies (7). By using Lemma 5 , we can obtain that (43) holds. So, it concludes that (42) holds if (43) holds, which completes the proof.

Combining Lemma 6 and Theorem 9, we have the following theorem.

Theorem 10. Consider system (1), and let $\gamma>0$ and $\delta_{a}>$ 0 be given constants; there exists a quantized fault detection observer (2) such that error closed-loop system (9) is stable and with the finite frequency performance

$$
\sigma_{\min } G_{r f}\left(e^{j \theta}\right)>\gamma, \quad \forall \theta \in\left[\vartheta_{2}, \vartheta_{2}\right]
$$

if there exist matrix variables $\Gamma, V_{b i}, V_{c i}, i=1, \ldots, 6, \bar{X}=$ $\left[\begin{array}{ll}X & 0 \\ 0 & X\end{array}\right]>0, P=\left[\begin{array}{ll}P_{11} & P_{12} \\ P_{21} & P_{22}\end{array}\right]$, and $Q=\left[\begin{array}{ll}Q_{11} & Q_{12} \\ Q_{12}^{T} & Q_{22}\end{array}\right]>0$ and scalars $\vartheta_{1}, \vartheta_{2}, \varepsilon_{1}$, and $\varepsilon_{2}$ such that the following inequalities hold:

$$
\begin{gathered}
{\left[\begin{array}{cccccc}
-X & * & * & * & * & * \\
0 & -X & * & * & * & * \\
X A-\Gamma^{T} C & 0 & -X & * & * & * \\
-X B_{2} K & X A+X B_{2} K & 0 & -X & * & * \\
0 & 0 & -\Gamma & 0 & -\varepsilon_{1} I & * \\
0 & \delta_{a} \varepsilon_{1} C & 0 & 0 & 0 & -\varepsilon_{1} I
\end{array}\right]<0} \\
{\left[\begin{array}{ccc}
\Lambda & F_{a 1} & \delta_{a} \varepsilon_{2} F_{a 2}^{T} \\
* & -\varepsilon_{2} I & 0 \\
* & * & -\varepsilon_{2} I
\end{array}\right]<0,}
\end{gathered}
$$

where $\delta_{a}=\max \left\{\delta_{i}, i=1, \ldots, y\right\}$ and $\Lambda$ is defined by (38), and the observer gain $L$ is obtained as $L^{T}=\Gamma X^{-1}$.

Remark 11. Note that, due to the existence of the unknown controller gain, the conditions given in Theorem 10 are not convex. In order to solve this problem, we design a controller gain by state feedback method as follows:

$$
\left[\begin{array}{cc}
-X & X A^{T}+\bar{K}^{T} B_{2}^{T} \\
A X+B_{2} \bar{K} & -X
\end{array}\right]<0,
$$

for $X>0$, and the controller gain is given as $K=\bar{K} X^{-1}$. Then use the state feedback controller gain as the initial value to obtain the observer gain $L$. So the following algorithm is given.

Algorithm 12. Let $\delta_{a}>0$ be given scalars and $\zeta>0$ a given small constant specifying a convergence criterion.

Step 1. By (51), we obtain the initial solutions $K^{\text {ini }}$; go to Step 2.
Step 2. Letting $K=K^{\mathrm{ini}}$,

$$
\begin{array}{cc}
\max & \gamma \\
\text { s.t. } & (50) \text {, }
\end{array}
$$

we obtain $\Gamma, X, V_{b i}, i=1, \ldots, 6$, and $\gamma_{\text {ini }}$; then go to Step 3 .

Step 3. Let $X_{\mathrm{ini}}=X, V_{b i}^{\mathrm{ini}}=V_{b i}, i=1, \ldots, 6$,

$$
\begin{array}{cc}
\max & \gamma \\
\text { s.t. } & (50) .
\end{array}
$$

$\gamma$ and $K$ are obtained. Then if $\left\|\gamma-\gamma_{\text {ini }}\right\|<\zeta$, stop, and $L^{T}=$ $\Gamma X^{-1}$, else, let $K=K$ and $\gamma_{\text {ini }}=\gamma$; return to Step 2 .

Remark 13. $\varepsilon_{1}, \varepsilon_{2}$ in (50) can be obtained by searching method to guarantee that the performance $\gamma$ is maximum.

\section{Example}

In this section, an example is given to illustrate the effectiveness of the developed theory. Consider a linear system of form (1) with

$$
\begin{gathered}
A=\left[\begin{array}{ccc}
0.9673 & 0 & 0.12 \\
0.0293 & 0.8763 & -0.4 \\
0.0259 & 0 & 0.9032
\end{array}\right], \\
B_{1}=\left[\begin{array}{cc}
0.1 & 0 \\
0.5 & 0.06 \\
-0.2 & 0.15
\end{array}\right], \quad B_{2}=\left[\begin{array}{cc}
1 & 0.04 \\
0 & 0.3 \\
0 & 0.05
\end{array}\right], \\
D_{1}=\left[\begin{array}{cc}
0.1 & 0 \\
0.5 & 12
\end{array}\right], \quad D_{2}=\left[\begin{array}{cc}
1 & 0 \\
0.5 & 0.1
\end{array}\right], \\
C=\left[\begin{array}{ccc}
0.1 & 0 & 0 \\
1 & 1 & 0
\end{array}\right] .
\end{gathered}
$$

For this example, set the quantization densities as $\rho_{1}=\rho_{2}=$ 0.91 . Assume that the frequency range of faults is known as $\theta \in[0,0.5]$. Let $\varepsilon_{1}=0.9899, \varepsilon_{2}=1.1299$; by Algorithm 12, we obtain the fault sensitivity performance index $\gamma=1.2131$, and, correspondingly, the quantized fault detection observer gain matrix $L_{\text {finite }}$ is obtained as

$$
L_{\text {finite }}=\left[\begin{array}{cc}
0.1452 & 0.0541 \\
-0.0145 & -0.2156 \\
-0.5421 & 0.3741
\end{array}\right] .
$$

In order to study the effects of fault on residual of the quantized detection observer, the fault signal is selected as

$$
f(k)= \begin{cases}{\left[\begin{array}{ll}
1 & 0.9
\end{array}\right]^{T},} & k \geq 20, \\
{\left[\begin{array}{ll}
0 & 0
\end{array}\right]^{T},} & \text { otherwise. }\end{cases}
$$

Using the observer gain matrix $L_{\text {finite }}$ given in (48), the two residual outputs are denoted by the solid lines of Figures 1 and 2 , respectively, from which we can see that the faulty cases are well discriminated from the fault free cases in presence of the disturbance effects. 


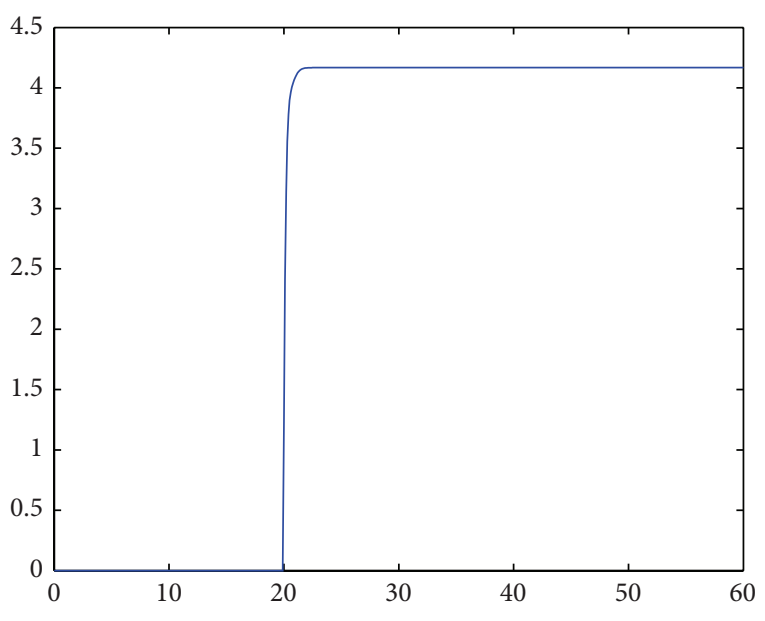

FIGURE 1: Residual output signal.

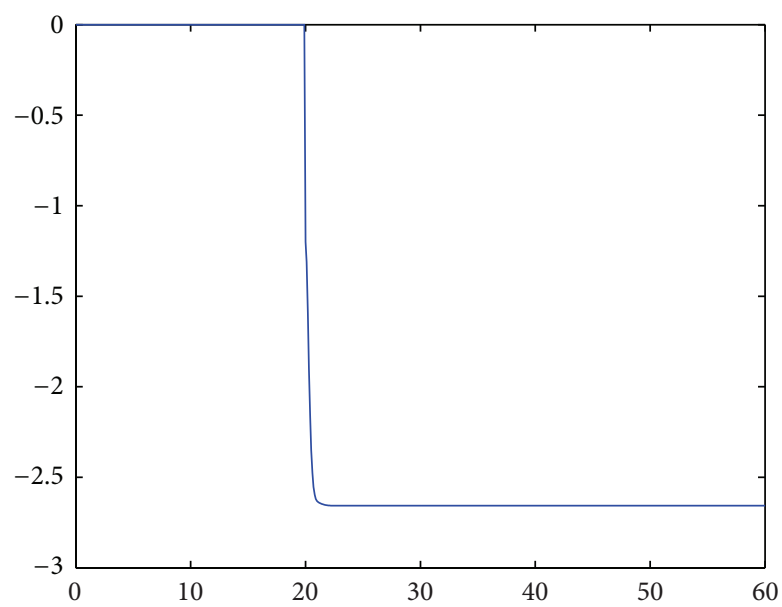

FIGURE 2: Residual output signal.

\section{Conclusion}

This paper considers the fault detection problem in the finite frequency domain for networked control systems with signal quantization. A quantized fault detection observer is designed by employing a performance index which is used to increase the fault sensitivity in finite frequency domain. By using the logarithmic quantizer method, the quantized measurement signals are dealt with by utilizing the sector bound method, in which the quantization error is treated as sector-bounded uncertainty. Further, By using the GKYP Lemma, an iterative LMI-based optimization algorithm is developed to design the quantized fault detection observer. Finally, a numerical example is given to illustrate the the effectiveness of the proposed method.

\section{Conflict of Interests}

One of the authors declares that there is no conflict of interests regarding the publication of this paper.

\section{Acknowledgments}

This work is partially supported by the Funds of National Science of China (Grant nos. 61104106 and 61104029), the Natural Science Foundation of Liaoning Province (Grant nos. 201202156 and 2013020144), and by Program for Liaoning Excellent Talents in University (LNET) (LJQ2012100).

\section{References}

[1] D. F. Delchamps, "Stabilizing a linear system with quantized state feedback," Institute of Electrical and Electronics Engineers, vol. 35, no. 8, pp. 916-924, 1990.

[2] N. Elia and S. K. Mitter, "Stabilization of linear systems with limited information," Institute of Electrical and Electronics Engineers, vol. 46, no. 9, pp. 1384-1400, 2001.

[3] M. Fu and L. Xie, "The sector bound approach to quantized feedback control," Institute of Electrical and Electronics Engineers, vol. 50, no. 11, pp. 1698-1711, 2005.

[4] H. Gao and T. Chen, " $H_{\infty}$ estimation for uncertain systems with limited communication capacity," Institute of Electrical and Electronics Engineers, vol. 52, no. 11, pp. 2070-2084, 2007.

[5] M. Fu and L. Xie, "Quantized feedback control for linear uncertain systems," International Journal of Robust and Nonlinear Control, vol. 20, no. 8, pp. 843-857, 2010.

[6] X. Yao, L. Wu, and W. X. Zheng, "Quantized $H_{\infty}$ filtering for Markovian jump LPV systems with intermittent measurements," International Journal of Robust and Nonlinear Control, vol. 23, no. 1, pp. 1-14, 2013.

[7] Y. Niu, T. Jia, X. Wang, and F. Yang, "Output-feedback control design for NCSs subject to quantization and dropout," Information Sciences, vol. 179, no. 21, pp. 3804-3813, 2009.

[8] W.-W. Che and G.-H. Yang, "Quantised $H_{\infty}$ filter design for discrete-time systems," International Journal of Control, vol. 82, no. 2, pp. 195-206, 2009.

[9] W.-W. Che, J.-L. Wang, and G.-H. Yang, "Quantised $H_{\infty}$ filtering for networked systems with random sensor packet losses," IET Control Theory \& Applications, vol. 4, no. 8, pp. 1339-1352, 2010.

[10] W. W. Che, J. L. Wang, and G. H. Yang, " $H_{\infty}$ control for networked control systems with limited communication," European Journal of Control, vol. 18, no. 2, pp. 103-118, 2012.

[11] R. Isermann, "Supervision, fault-detection and fault-diagnosis methods-an introduction," Control Engineering Practice, vol. 5, no. 5, pp. 639-652, 1997.

[12] J. J. Gertler, Fault Detection and Diagnosis in Engineering Systems, Marcel Dekker, New York, NY, USA, 1998.

[13] I. M. Jaimoukha, Z. Li, and V. Papakos, "A matrix factorization solution to the $H_{-} / H_{\infty}$ fault detection problem," Automatica, vol. 42, no. 11, pp. 1907-1912, 2006.

[14] M. Zhong, S. X. Ding, B. Tang, P. Zhang, and T. Jeinsch, "An LMI approach to robust fault detection filter design for discrete-time systems with model uncertainty," in Proceedings of the 40th IEEE Conference on Decision and Control (CDC '01), pp. 3613-3618, December 2001.

[15] J. Chen and R. Patton, Robust Model-Based Fault Diagnosis for Dynamic Systems, Kluwer Academic, Dordrecht, The Netherlands, 1999.

[16] G. Tao, S. M. Joshi, and X. Ma, "Adaptive state feedback and tracking control of systems with actuator failures," Institute of 
Electrical and Electronics Engineers, vol. 46, no. 1, pp. 78-95, 2001.

[17] F. Liao, J. L. Wang, and G. H. Yang, "Reliable robust flight tracking control: an LMI Approach," IEEE Transactions on Control Systems Technology, vol. 10, pp. 76-89, 2002.

[18] H. Wang, J. Wang, and J. Lam, "An optimization approach for worst-case fault detection observer design," in Proceedings of the American Control Conference (AAC '04), pp. 2475-2480, July 2004.

[19] H. Wang, J. Wang, J. Liu, and J. Lam, "Iterative LMI approach for robust fault detection observer design," in Proceedings of the 42nd IEEE Conference on Decision and Control, pp. 1974-1979, December 2003.

[20] H. Wang and G.-H. Yang, "A finite frequency domain approach to fault detection for linear discrete-time systems," International Journal of Control, vol. 81, no. 7, pp. 1162-1171, 2008.

[21] Z. Mao, B. Jiang, and P. Shi, "Protocol and fault detection design for nonlinear networked control systems," IEEE Transactions on Circuits and Systems II, vol. 56, no. 3, pp. 255-259, 2009.

[22] D. Huang and S. K. Nguang, "Robust fault estimator design for uncertain networked control systems with random time delays: an ILMI approach," Information Sciences, vol. 180, no. 3, pp. 465-480, 2010.

[23] T. Iwasaki and S. Hara, "Generalized KYP lemma: unified frequency domain inequalities with design applications," Institute of Electrical and Electronics Engineers, vol. 50, no. 1, pp. 41-59, 2005.

[24] I. R. Petersen, "A stabilization algorithm for a class of uncertain linear systems," Systems \& Control Letters, vol. 8, no. 4, pp. 351357, 1987. 


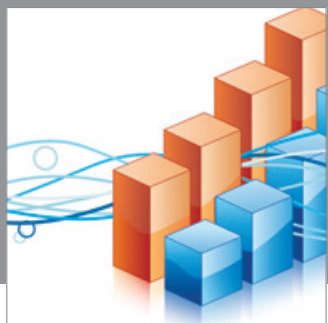

Advances in

Operations Research

mansans

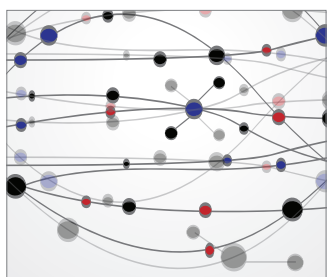

The Scientific World Journal
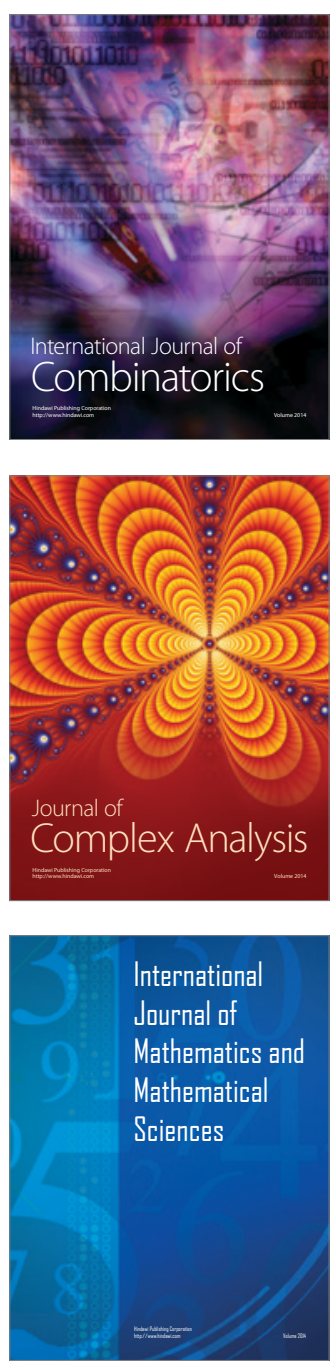
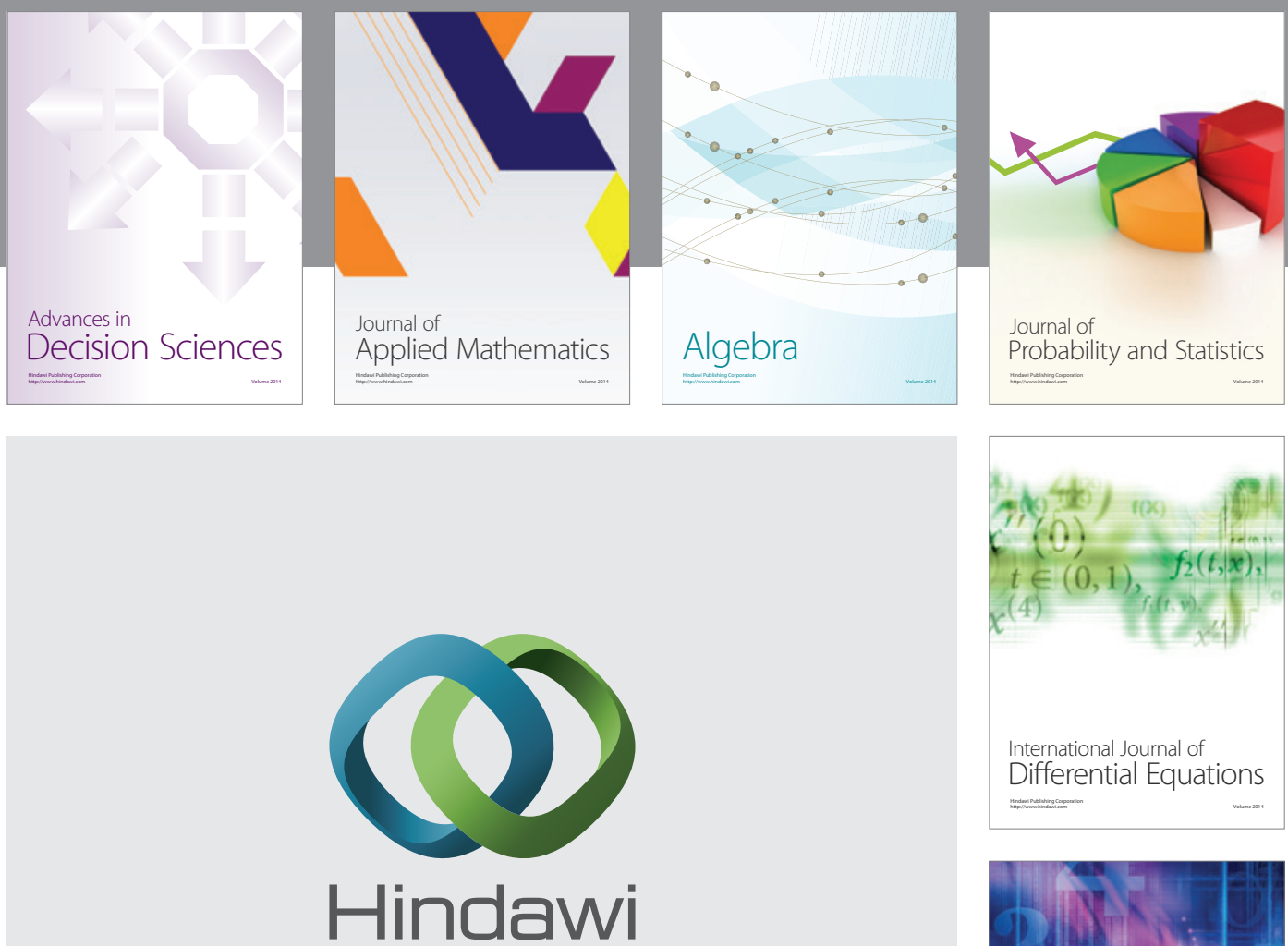

Submit your manuscripts at http://www.hindawi.com
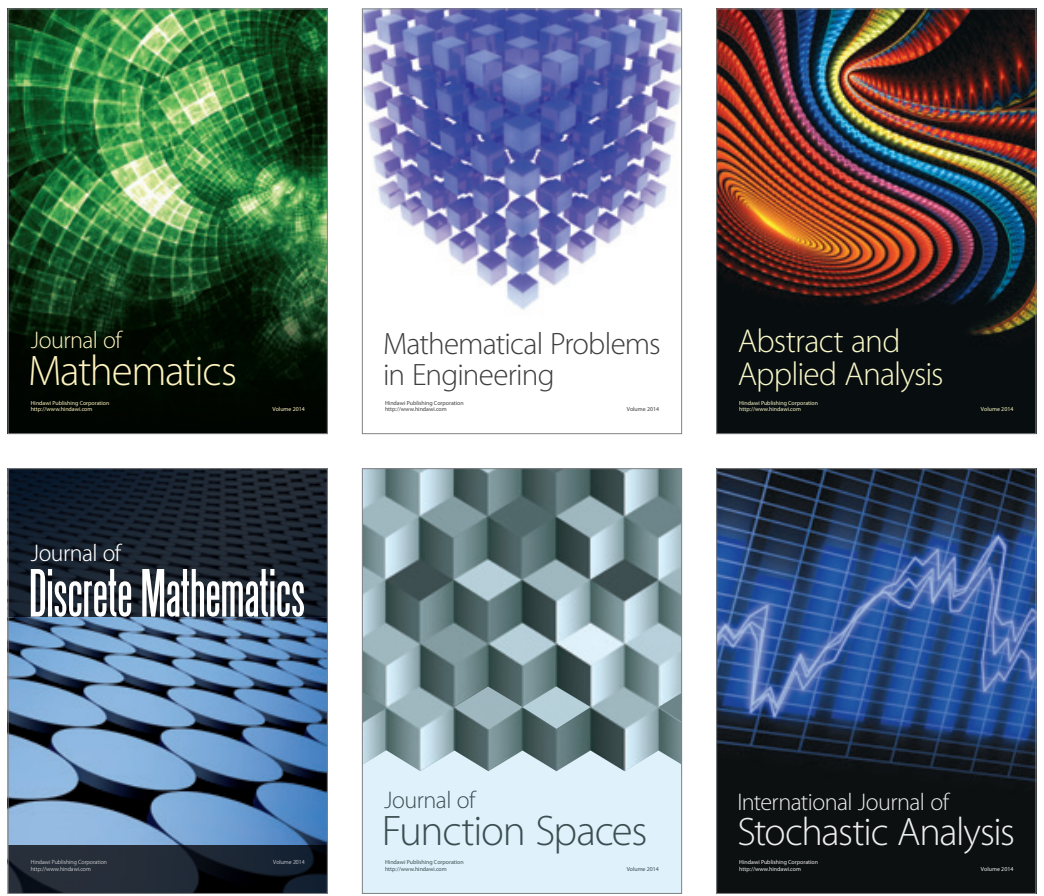

Journal of

Function Spaces

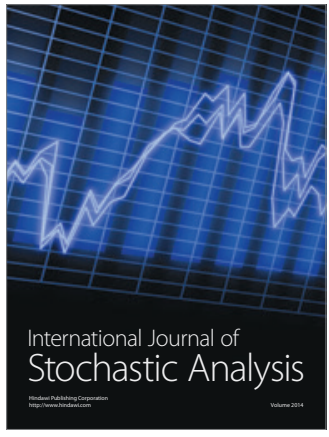

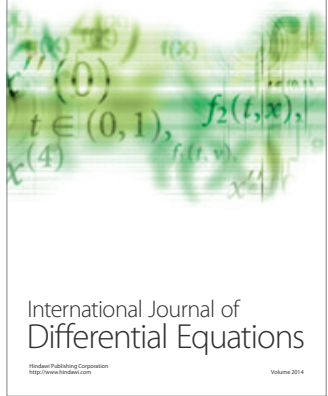
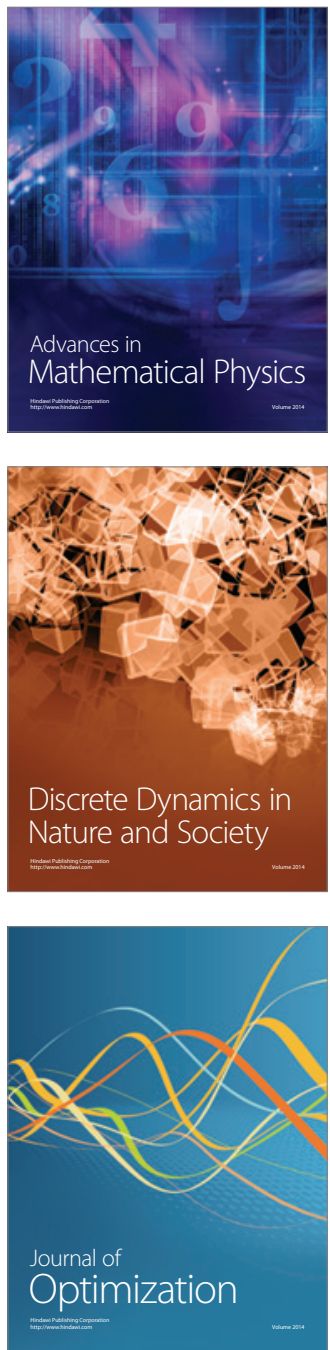